\title{
Knockdown of damage-specific DNA binding protein 1 (DDB1) enhances the HBx-siRNA-mediated inhibition of HBV replication
}

\author{
Kai-Fu Tang ${ }^{\text {a,* }}$, Jing Xie ${ }^{\text {a }}$, Min Chen ${ }^{\text {a }}$, Qi Liu ${ }^{\text {a }}$, Xi-Yuan Zhou ${ }^{b}$, Weiqun Zeng ${ }^{\text {a }}$, \\ Ai-Long Huang ${ }^{\mathrm{a}}$, Guo-qing Zuo ${ }^{\mathrm{c}}$, Yan Wang ${ }^{\mathrm{d}}$, Rong Xiang ${ }^{\mathrm{e}}$, Hong Ren ${ }^{\mathrm{a}, * *}$ \\ ${ }^{a}$ Key Laboratory of Molecular Biology for Infectious Diseases, Ministry of Education, Institute for Viral Hepatitis, The Second Affiliated Hospital, \\ Chongqing University of Medical Sciences, 74\# Linjiang Road, Chongqing 400010, PR China \\ ${ }^{\mathrm{b}}$ Department of Ophthalmology, The Second Affiliated Hospital, Chongqing Medical University, Chongqing 400010, PR China \\ ${ }^{c}$ Department of Gastroenterology, The Second Affiliated Hospital, Chongqing Medical University, Chongqing 400010, PR China \\ ${ }^{\mathrm{d}}$ The Institute of Psychology, Chinese Academy of Sciences (CAS), Beijing 100101, PR China \\ e Department of Immunology, The Scripps Research Institute, 10550 North Torrey Pines Road, La Jolla, CA 92037, USA
}

Received 25 March 2007; revised 2 September 2007; accepted 12 November 2007

\begin{abstract}
Recent studies have demonstrated that the effect of inhibition of HBV replication can be achieved by RNA interference (RNAi) at both the cellular and organismal levels. However, HBV replication cannot be completely inhibited by this method. To completely inhibit HBV replication, new strategies for improving the inhibition efficacy of HBV-specific siRNAs are needed. In this study, we demonstrated that knockdown of damage-specific DNA binding protein 1(DDB1), a protein involved in nucleotide-excision repair and HBV replication, significantly enhanced the HBx-siRNA-mediated inhibition of HBV replication. Although knockdown of DDB1 may be toxic to normal liver cells, our results indeed suggest a new direction to enhance the efficacy of HBV-siRNA-mediated inhibition of HBV replication.
\end{abstract}

(C) 2007 The International Association for Biologicals. Published by Elsevier Ltd. All rights reserved.

Keywords: Hepatitis B virus; RNAi; Damage-specific DNA binding protein 1

\section{Introduction}

Hepatitis B virus (HBV) infection is a major worldwide health problem, particularly in Asia. Globally, approximately 350 million people are chronic carriers of $\mathrm{HBV}$ and they have a $15-25 \%$ risk of dying from end-stage cirrhosis and hepatocellular carcinoma (HCC) [1]. Licensed treatments for HBV infection, which include interferon- $\alpha$, and nucleoside (lamivudine) and nucleotide (adefovir) analogues, produce a long-term response only in a minority of chronic carriers [2]. The continuing search for an effective therapy to prevent life-threatening complications thus remains a priority.

\footnotetext{
* Corresponding author. Tel.: +86 1345203 7049; fax: +86 2363822696 .

** Corresponding author.

E-mail addresses: tangkaifu@hotmail.com, tang_kaifu@yahoo.com.cn (K.-F. Tang), renhong0531@vip.sina.com (H. Ren).
}

RNA interference (RNAi) is a natural process in eukaryotic cells by which double-stranded RNA initiates and directs the degradation of homologous mRNA [3]. This RNA silencing mechanism was first described in Caenorhabditis elegans [4]. RNAi technology has emerged not only as an extremely powerful tool for functional genomic studies, but also as a potentially useful method for developing specific gene-silencing therapeutics, especially for the treatment of viral diseases. Several groups have demonstrated the efficacy of RNAi against HBV replication. Chemically synthesized siRNA, expressed short hairpin RNA (shRNA), and endoribonuclease-prepared siRNA (esiRNA) have been used to inhibit HBV replication in vitro and in vivo. These pioneering studies have demonstrated great possibilities for treating HBV infection [5-10]. However, HBV replication can not be completely inhibited by these methods. To completely inhibit HBV replication, new strategies for improving the inhibition efficacy of HBV-specific siRNAs are 
needed. Although $\mathrm{Wu}$ and colleagues showed that the cocktail of HBs-siRNA and HBx-siRNA is more potent in inhibiting $\mathrm{HBV}$ replication than any single siRNA, the maximum inhibition efficacy is still $<90 \%$ [7].

Various ubiquitous and liver-enriched transcription factors, such as HNF4, RXR $\alpha$, PPAR $\alpha$, COUP-TF1, DDB1, and HNF3, have been shown to modulate HBV transcription [11]. We propose that modulation of the activity of these factors will transcriptionally inhibit $\mathrm{HBV}$ replication, and hence will enhance the activity of HBV-specific siRNAs, which post-transcriptionally repress HBV replication.

DDB1 is the large subunit of DNA damage-binding protein, which is a heterodimer composed of a large and a small subunit. This protein functions in nucleotide-excision repair $[12,13]$. DDB1 physically interacts with Hepatitis B virus X protein and regulates its turnover $[14,15]$. HBx is a pleiotropic protein, which can stimulate viral replication by activating HBV transcription $[16,17]$ or by enhancing the reverse transcription activity of the viral polymerase [18]. Recently, Leupin and colleagues showed that an interaction with DDB1 is also needed for $\mathrm{HBx}$ to stimulate $\mathrm{HBV}$ transcription, and that knockdown of DDB1 significantly compromises HBV transcription [19]. Therefore, we reasoned that knockdown of DDB1 will not only repress HBV transcription, but also destabilize HBx protein, and hence enhance the antiviral activity of HBX-siRNA. To test this hypothesis, we transfected HepG2.2.15 cells with HBx-siRNA, DDB1-siRNAs, or both. Our results showed that knockdown of DDB1 compromised HBV replication, and co-transfection with DDB1-siRNAs and $\mathrm{HBX}$-siRNA significantly enhanced the antiviral activity of HBx-siRNA.

\section{Materials and methods}

\subsection{Construction of siRNAs}

siRNAs were prepared using a Silencer siRNA Construction Kit (Ambion, Austin, TX). Briefly, sense and antisense oligonucleotide templates were annealed with $\mathrm{T} 7$ promoter primer, and then filled in with Klenow DNA polymerase. Using the filled-in dsDNAs as templates, sense and antisense RNAs were transcribed with T7 RNA polymerase. Sense and antisense RNAs were annealed and the single stranded leader sequences were removed by digesting the dsRNA with a single-strand specific ribonuclease. The HBx-siRNA targeting sequence 5'-GCACUUCGCUUCACCUCUG-3' was described by Morrissey [10]. The DDB1-siRNA1 targeting sequence 5'-CCUGUUGAUUGCCAAAAAC- $3^{\prime}$ was described by $\mathrm{Hu}$ [20]. The DDB1-siRNA2 targeting sequence was: $5^{\prime}$-GCAAG GACCUGCUGUUUAU- $3^{\prime}$. The control siRNA target sequence was $5^{\prime}$-AAUUCUCCGAACGUGUCACGU-3', which had no homology in the human genome.

\subsection{Cell culture, transfection and growth curves}

HepG2.2.15 cells are developed by transfection of the human HCC cell line HepG2 with a plasmid containing two head-to-tail dimers of the HBV genome, and they can constitutively secrete $\mathrm{HBsAg}, \mathrm{HBeAg}$, and Dane-like particles [21]. HepG 2.2.15 and L02 cells were cultured in RPMI 1640 medium supplemented with $10 \%$ fetal bovine serum (FBS). HepG2.2.15 cells were also supplemented with $200 \mu \mathrm{g} / \mathrm{mL}$ G418. All cultures were maintained at $37^{\circ} \mathrm{C}$ in a moist atmosphere containing $5 \% \mathrm{CO}_{2}$ in the air. Transfections were carried out using siPORT NeoFX (Ambion) according to the manufacturer's instructions. Briefly, cells were trypsinized and plated in 6-well plates at $4 \times 10^{5}$ cells per well for $30 \mathrm{~min}$ before transfection. Transfection complexes were prepared in Opti-mem serum-free medium (Invitrogen, CA) by mixing $5 \mu \mathrm{L}$ of siPORT NeoFX Transfection Reagent and $10 \mathrm{nM}$ of siRNA. The cells and medium were harvested $72 \mathrm{~h}$ after transfection. For the growth curves, shown in Fig. 4, cells transfected with different siRNAs were collected every $24 \mathrm{~h}$. The cell number was assayed in triplicate, and each growth curve represents the mean of three independent experiments.

\subsection{Western blotting analysis}

Cells were collected on day 3 post-transfection, washed in phosphate-buffered saline (PBS), and lysed in RIPA buffer. Total protein was measured using the Bradford protein assay (Beyotime, Jiangsu, China). Samples were denatured at $100{ }^{\circ} \mathrm{C}$ for $5 \mathrm{~min}$. Equal amounts of total protein were loaded to each well for electrophoresis in $12 \%$ SDS-polyacrylamide gels and then transferred to polyvinylidine fluoride microporous membranes (Millipore Corporation, Billerica, MA). Membranes were then incubated with primary antibody, followed by incubation with horseradish peroxidase-linked secondary antibodies. The primary antibodies included antiHBx (Chemicon, CA) and anti-GAPDH (Kangchen Biotech, Shanghai, China).

\subsection{Quantitative assay of viral proteins}

The viral proteins of hepatitis B surface antigen (HBsAg) and e antigen ( $\mathrm{HBeAg}$ ) in culture medium from transfected cells were measured using the Abbott Microparticle Enzyme Immunoassay kit (Abbott Diagnostics, IL) according to the manufacturer's instructions.

\subsection{Real-time RT-PCR analysis}

Total RNA was prepared using TRIZOL (Invitrogen, CA) and reverse-transcribed using MMLV reverse transcriptase (TOYOBO Bio-Technology. Co., Ltd, Shanghai, China) according to the manufacturer's protocol. The resulting cDNA was amplified with primer pairs in triplicate, and amplification was monitored using SYBRGreen chemistry. Primer sequences (forward and reverse, respectively) were as follows:

GAPDH: ATGACATCAAGAAGGTGGTG and CATACC AGGAAATGAGCTTG;

DDB1: ATCATCCGGAATGGAATTGGAA and TCAGA CCGCAGTGGCCATAA. 
The level of DDB1 expression was measured using $\mathrm{Ct}$ (threshold cycle). The $\mathrm{Ct}$ is the fractional cycle number at which the fluorescence of each sample passes the fixed threshold. The $\Delta \Delta \mathrm{Ct}$ method for relative quantitation of gene expression was used to determine DDB1 expression levels. The $\Delta \mathrm{Ct}$ was calculated by subtracting the $\mathrm{Ct}$ of the GAPDH mRNA from the Ct of the DDB1 mRNA. The $\Delta \Delta \mathrm{Ct}$ was calculated by subtracting the $\Delta \mathrm{Ct}$ of the reference sample (i.e., cells transfected with control siRNA) from the $\Delta \mathrm{Ct}$ of each sample. The fold change was generated using the equation $2^{-\Delta \Delta \mathrm{Ct}}$. The relative DDB1 mRNA level in each sample was expressed as the percentage of the mRNA level in the cells transfected with control siRNA, which was defined as $100 \%$.

\subsection{Isolation of nuclear cccDNA}

Low-molecular-weight nucleic acids comprising HBV cccDNA and host mitochondrial DNA were isolated as described [22]. Cell layers were lysed by the addition of $400 \mu \mathrm{l}$ of lysis buffer (50 mM Tris- $\mathrm{HCl}[\mathrm{pH} 8] / 1 \mathrm{mM}$ EDTA/0.2\% Nonidet P-40/0.15 M NaCl). The nuclei were pelleted by microcentrifugation for $1 \mathrm{~min}$ and then lysed by the addition of $400 \mu \mathrm{l}$ of nuclei lysis buffer (6\% [vol/vol] SDS/0.1 M $\mathrm{NaOH})$. The reaction was mixed thoroughly and incubated for $30 \mathrm{~min}$ at $37^{\circ} \mathrm{C}$ with occasional mixing. The alkaline lysate was neutralized by the addition of $200 \mu \mathrm{l}$ of neutralization buffer (3 M KAc [pH 4.8]) and centrifuged for $15 \mathrm{~min}$ at $4{ }^{\circ} \mathrm{C}$ at $12,000 \times \mathrm{g}$. The supernatant was extracted with water-saturated phenol $(\mathrm{pH} 4.5)$ followed by chloroform extraction, and nucleic acids were precipitated with isopropanol in the presence of $10 \mu \mathrm{g}$ of Escherichia coli tRNA. The precipitate was dissolved in $50 \mu \mathrm{l}$ of TE $(10 \mathrm{mM}$ Tris $-\mathrm{HCl}$ [pH 7.5]/ $1 \mathrm{mM}$ EDTA).

\subsection{Real-time PCR}

Medium HBV DNA was extracted from $100 \mu \mathrm{L}$ transfected cell culture supernatant and quantified using a HBV real-time PCR kit (Fuxing Biotech, Shanghai, China).

Nuclear CCC HBV DNA was quantified by a modification of a previously described procedure [22]. Briefly, nuclear cccDNA was measured using an HBV real-time PCR kit (Fuxing Biotech). All samples were normalized to the signal generated from mitochondrial DNA. Mitochondrial DNA was amplified with a primer pair (CCG GAG AGT ATA TTG TTG AAG AG and CAC AGA AGC TGC CAT CAA GTA) in triplicate and amplification was monitored using SYBRGreen chemistry.

\section{Results}

\subsection{HBx-siRNA treatment suppresses HBV replication}

HepG 2.2.15 cells were transfected with HBx-siRNA; Western blotting verified that $\mathrm{HBx}$-siRNA significantly inhibited the expression of HBx protein (Figs. 1A,B). The HBsAg and $\mathrm{HBeAg}$ secreted into the culture medium were quantified by using a microparticle enzyme immunoassay on day 3 posttransfection. The HBsAg and HBeAg levels in the medium decreased to 56 and $47 \%$, respectively, in cells transfected with HBx-siRNA, in comparison with cells transfected with control siRNA (Fig. 2). The result of quantitative PCR indicated that the medium HBV DNA level and the nuclear HBV cccDNA level were decreased to 28 and $21 \%$, respectively, in cells transfected with $\mathrm{HBX}$-siRNA in comparison to cells transfected with control siRNA (Fig. 3).

\subsection{Knockdown of DDB1 destabilizes HBx protein}

To knock down DDB1, HepG 2.2.15 cells were transfected with DDB1-siRNA1 and collected 3 days after transfection. Real-time RT-PCR indicated that the level of DDB1 mRNA was significantly decreased and that co-transfection with HBx-siRNA did not interfere with the effect of DDB1-siRNA1 (Fig. 1C). The physical interaction between the DDB1 and HBx leads to an increase in the stability of $X$ protein [15]. Therefore, knockdown of DDB1 may destabilize HBx protein. Western blotting indeed showed that knockdown of DDB1 led to a reduction of $\mathrm{HBx}$ protein and in cells co-transfected with both HBx-siRNA and DDB1-siRNA1, the level of HBx protein was further decreased compared to that in cells transfected with HBx-siRNA alone (Fig. 1A). DDB1-siRNA2 also decreased the stability of HBx protein (Figs. 1B), which suggests that destabilization of HBx protein is not the off-target effect of DDB1-siRNAs.

\subsection{Knockdown of $D D B 1$ inhibits $H B V$ replication}

Leupin and colleagues showed that RNAi-mediated inhibition of DDB1 leads to a pronounced decrease in wild-type HBV replication [19]. To test whether HBV replication is also repressed by DDB1 knockdown in our system, medium HBsAg, HBeAg, HBV DNA, and nuclear HBV cccDNA were quantified. The levels of medium $\mathrm{HBsAg}, \mathrm{HBeAg}$, HBV DNA, and nuclear cccDNA decreased to 87, 86, 68, and $43 \%$, respectively, in cells transfected with DDB1siRNA1 in comparison to cells transfected with control siRNA. Similar results were obtained with DDB1-siRNA2 (Figs. 2 and 3).

\subsection{Knockdown of DDB1 enhances the HBx-siRNA- mediated inhibition of $H B V$ replication}

In order to test our hypothesis that knockdown of DDB1 will enhance the antiviral activity of HBx-siRNA, we co-transfected both DDB1-siRNA1 and HBx-siRNA into HepG 2.2.15 cells. As described above, the medium HBsAg, HBeAg, HBV DNA, and nuclear cccDNA levels in cells transfected with HBx-siRNA were 56, 47, 28, and $21 \%$, respectively, of the levels in cells transfected with control siRNA. In contrast, in cells co-transfected with DDB1-siRNA1 and HBx-siRNA, the medium of HBsAg, HBeAg, HBV DNA, and nuclear 

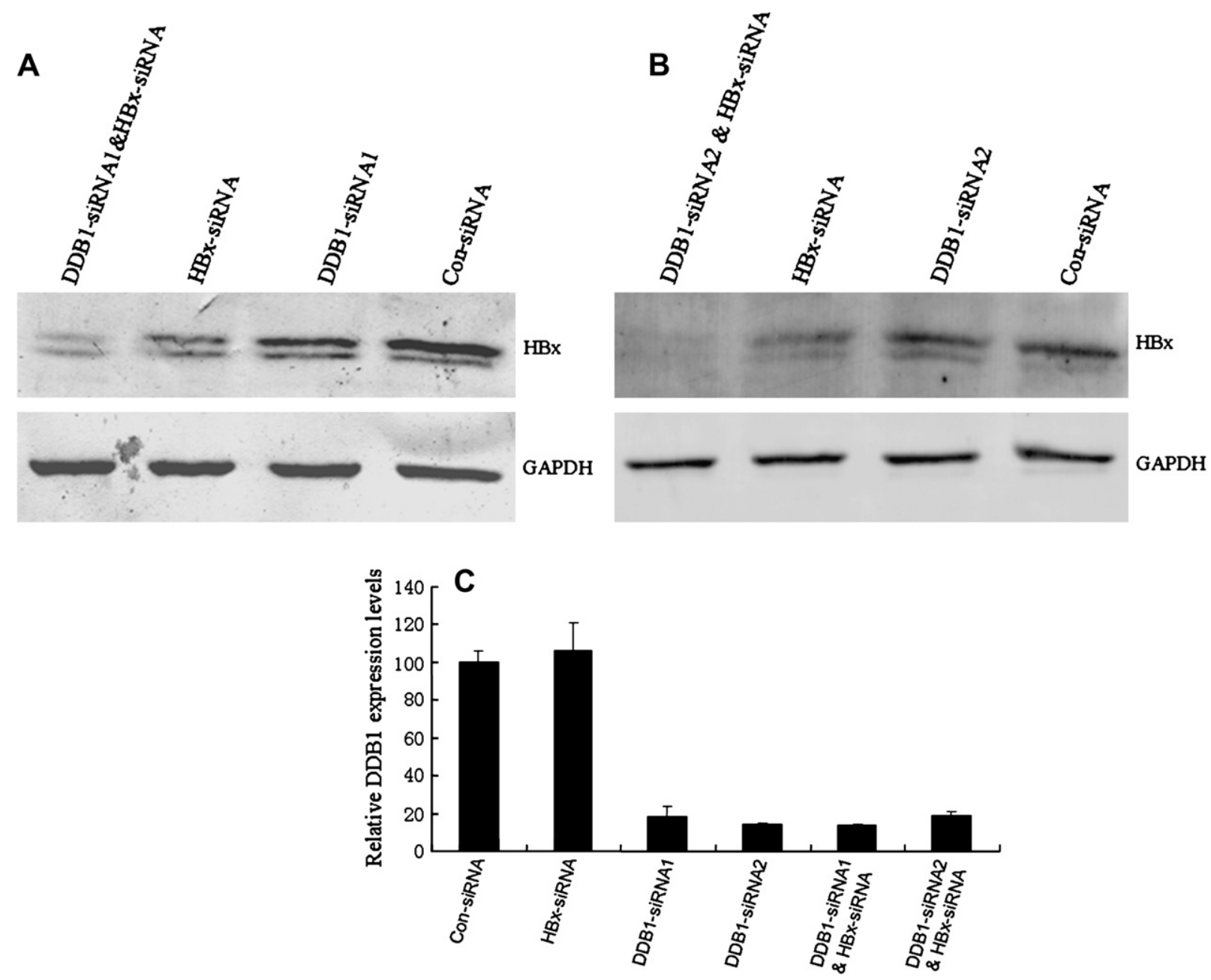

Fig. 1. (A, B) HBx level detected by Western blotting. HepG2.2.15 cells transfected with different siRNAs; the level of HBx protein was detected by Western blotting using anti-HBx antibody. (C) DDB1 mRNA was suppressed by DDB1-siRNAs. HepG2.2.15 cells were transfected with different siRNAs. Real time RT-PCR showed that DDB1 mRNA was reduced in cells transfected with DDB1-siRNAs and in cells co-transfected with both DDB1-siRNAs and HBx-siRNA; however, in cells transfected with control siRNA or HBx-siRNA, the DDB1 mRNA level was unaffected.

HBV cccDNA levels were $29,20,12$, and $8 \%$, respectively, of the levels in cells transfected with control siRNA. Similar results were observed with DDB1-siRNA2 (Figs. 2 and 3). These results indicate that knockdown of DDB1 can significantly enhance the inhibition efficiency of HBx-siRNA.

\subsection{Knockdown of DDB1 does not significantly affect proliferation of HepG.2.2.15 cells, but causes reduction of proliferation in LO2 cells}

DDB1 encodes a DNA damage-binding protein, which is involved in nucleotide-excision repair. Its defective activity causes the repair defect in the patients with xeroderma pigmentosum complementation group $\mathrm{E}$ (XPE) [12,13,23]. DDB1 is also required for the ubiquitination and degradation of the protooncogenic transcription factor c-Jun [24]. Therefore, knockdown of DDB1 may have toxic effects. To test this possibility, we examined whether knockdown of DDB1 could affect cell proliferation. Comparison of the growth curves of HepG2.2.15 cells transfected with different siRNAs indicated that knockdown of DDB1 did not significantly affect the growth of HepG2.2.15 cells. Furthermore, transfection with HBx-siRNA or co-transfection of DDB1-siRNAs along with HBx-siRNA did not affect cell growth (Fig. 4A). Taken together, these results indicated that knockdown of DDB1 in HepG2.2.15 cells does not lead to a reduction in cell proliferation. However, knockdown of DDB1 in the normal liver cell line, L02, resulted in reduced cell proliferation (Fig. 4B).

\section{Discussion}

DDB1 is a pleiotropic protein which has been implicated in DNA damage recognition, stimulation of HBV transcription, and association with a cullin-based ubiquitin ligase $[12,13,19,24]$. Previous studies have established the functional importance of an interaction between DDB1 and $\mathrm{HBx}$ in the stability of HBx protein [15]. In the present study, we show that knockdown of DDB1 destabilizes HBx. Furthermore, we demonstrated that knockdown DDB1 

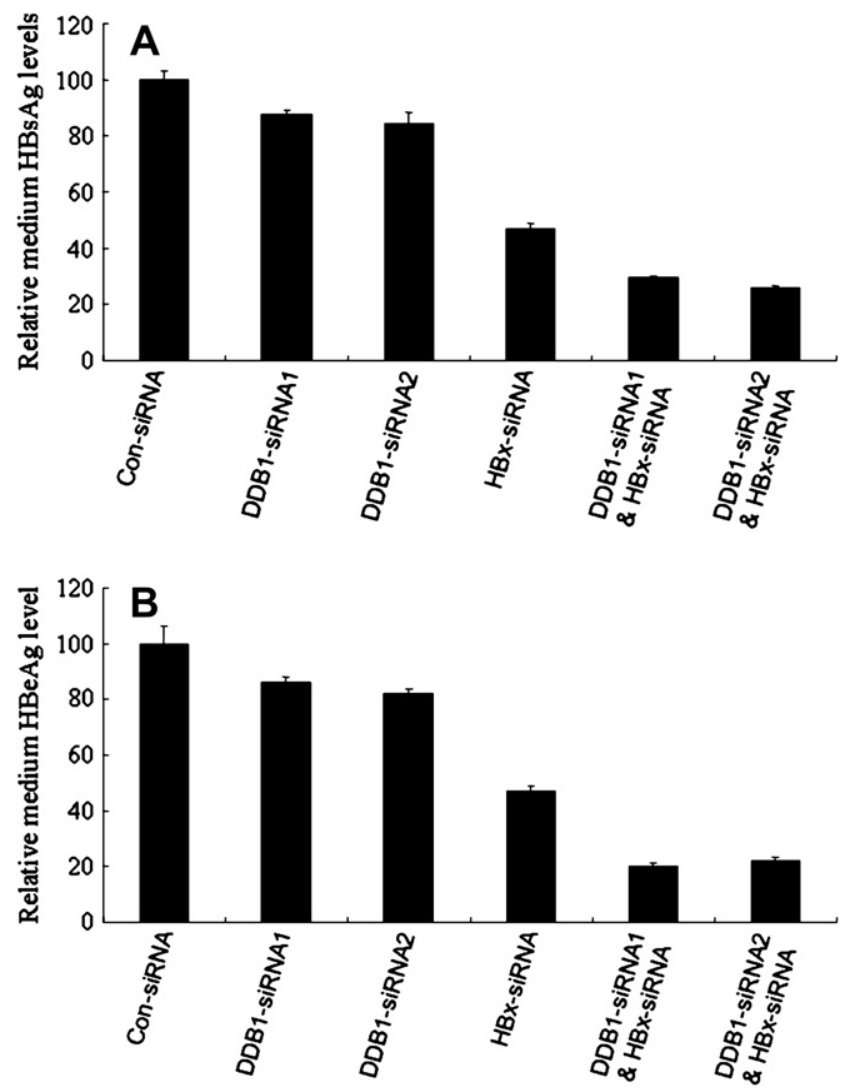

Fig. 2. The levels of HBsAg and HBeAg in the supernatant. HepG2.2.15 cells transfected with different siRNAs; the antigens in the supernatant were detected by microparticle enzyme immunoassay. The relative levels are normalized to the average of HBeAg and HBsAg in the medium from 2.2.15 cells transfected with control siRNA, which are defined as 100. Values are the mean \pm standard error of three independent experiments. (A) The medium HBsAg levels. (B) The medium HBeAg levels.

compromises HBV replication, which is consistent with the report suggesting an interaction with DDB1 is needed for HBx to stimulate HBV transcription [19].

Small interfering RNA (siRNA) offers a new tool with potential therapeutic application to combat HBV. However, published data indicate that $\mathrm{HBV}$ replication cannot be completely inhibited by this method [5-10]. Herein we have demonstrated that the levels of secreted $\mathrm{HBsAg}$, HBeAg, HBV DNA, and nuclear cccDNA in cells co-transfected with both HBx-siRNA and DDB1-siRNAs were decreased by a further 2-3 fold, compared to cells transfected with $\mathrm{HBx}$-siRNA alone; however, the level of DDB1 mRNA in cells co-transfected with both HBx-siRNA and DDB1-siRNAs was similar to cells transfected with DDB1-siRNA alone, which suggests that the transfection efficiency was not significantly different between cells transfected with single siRNA and cells co-transfected with both siRNAs. The concerted effect of HBx-siRNA and DDB1siRNA was not surprising because HBx-siRNA only mediates the cleavage of HBx mRNA; co-transfection of HBx-siRNA with DDB1-siRNAs not only degradates $\mathrm{HBx}$ transcripts, but also inhibits $\mathrm{HBx}$ transcription and destabilizes $\mathrm{HBx}$ protein.
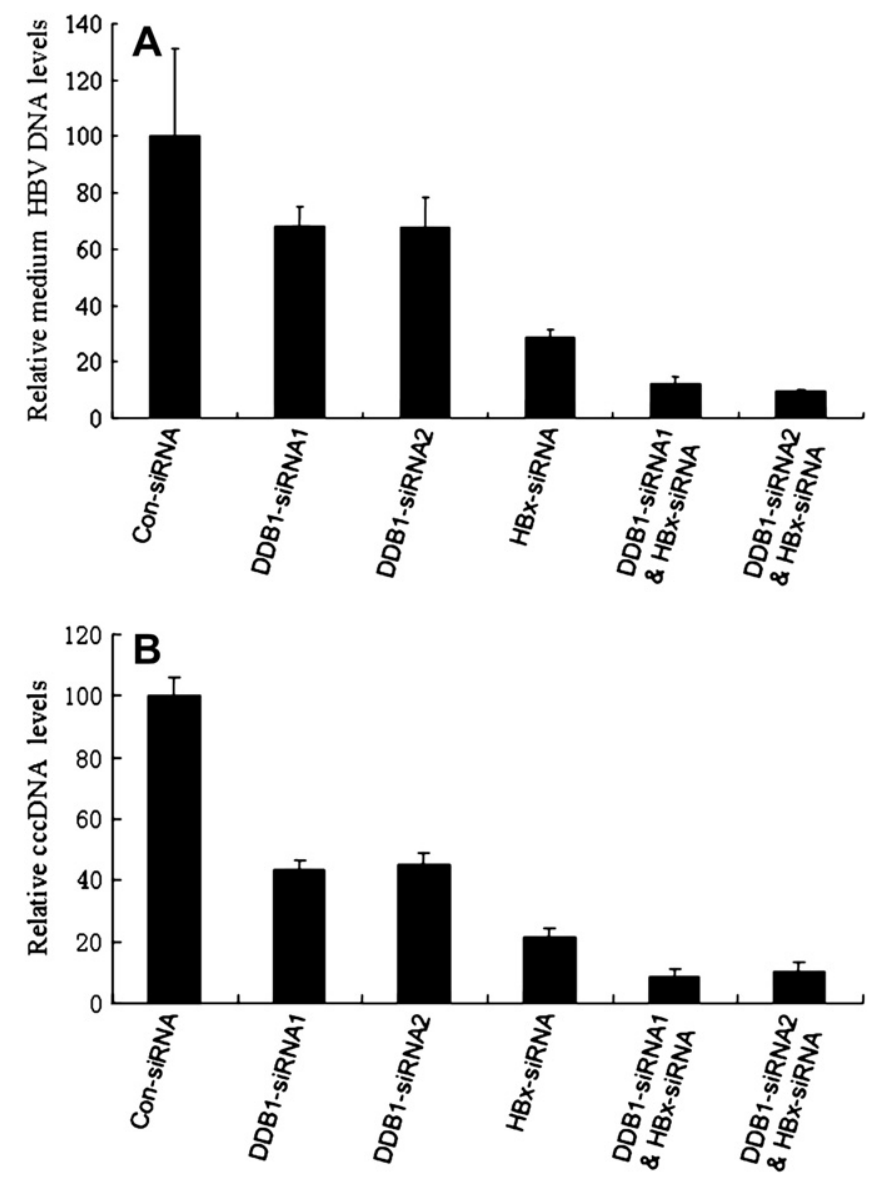

Fig. 3. Quantification of (A) medium HBV DNA; and (B) nuclear cccDNA. The levels of medium HBV DNA and nuclear cccDNA were quantified by real-time PCR. The relative levels are normalized to the average of medium HBV DNA or nuclear cccDNA from HepG2.2.15 cells transfected with control siRNA, which is defined as 100 . Values are the mean \pm standard error of three independent experiments.

DDB1 is a DNA damage protein that interacts with a variety of other proteins. Its defective activity causes the repair defect in the patients with xeroderma pigmentosum complementation group E (XPE) $[12,13,23]$. DDB1 is also essential for the ubiquitination and degradation of the protooncogenic transcription factor, c-Jun: ablation of DDB1 by RNA interference stabilizes c-Jun and increases c-Jun-activated transcription [24]. Therefore, knockdown of DDB1 may disrupt the physiologic functions of cells. However, knockdown of DDB1 did not significantly affect the growth of HepG2.2.15 cells in our study. This is not surprising, because Hepatitis B virus $X$ protein associates with DDB1 and induces cell death [25]. Knockdown of DDB1 may protect HBV-infected cells from apoptosis induced by HBx. Transfection of HBx-siRNA did not cause a reduction of proliferation in HepG2.2.15 cells, either. In contrast with HepG2.2.15 cells, the normal liver cell line, L02, showed reduced proliferation after transfection with DDB1-siRNAs. This result is consistent with the recent report that epidermis-specific deletion of DDB1 in mice leads to cell cycle arrest and apoptosis [26]. 

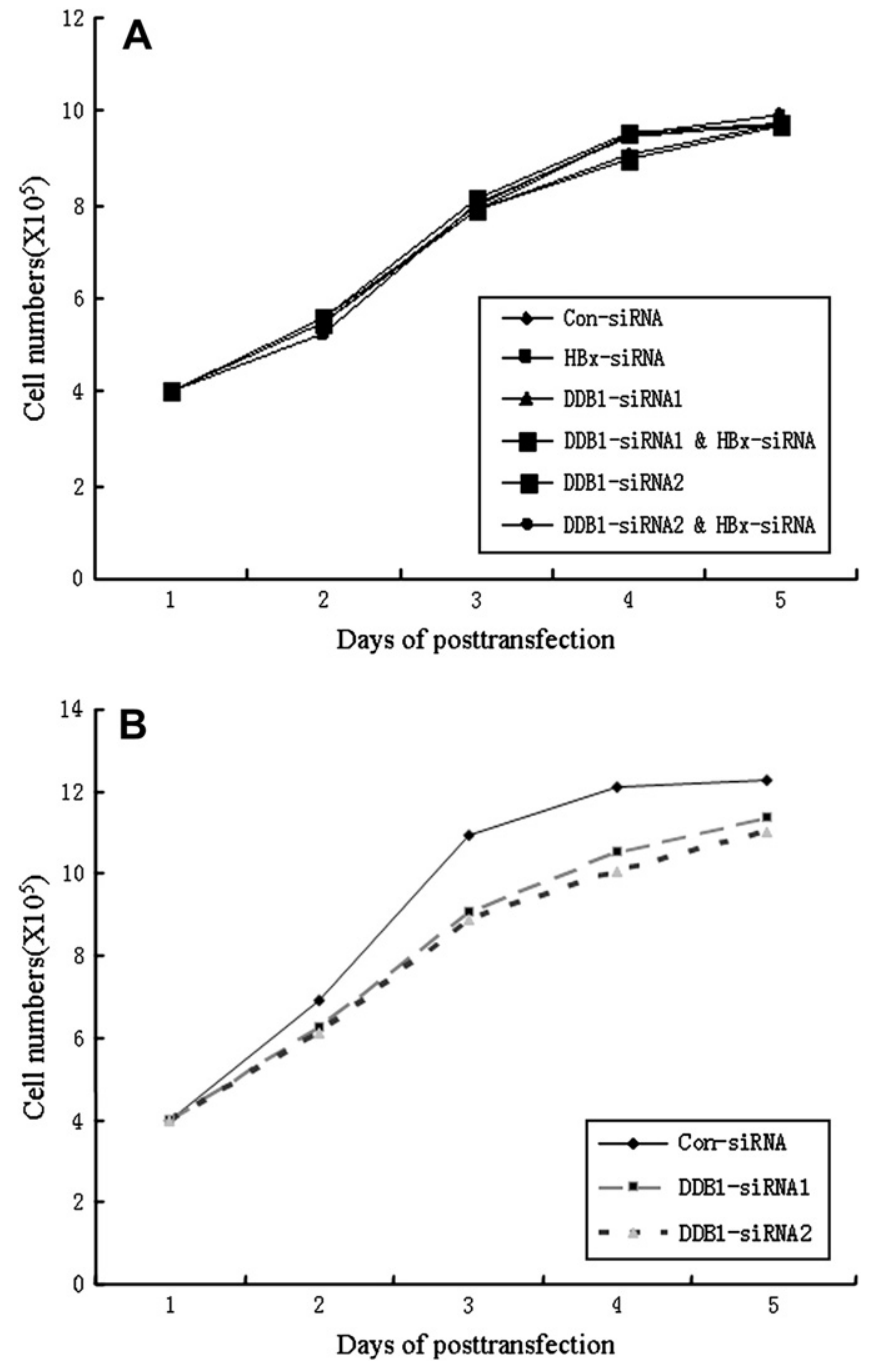

Fig. 4. Growth curve of HepG2.2.15 cells and L02 cells transfected with different siRNAs. (A) Knockdown of DDB1 did not significantly affect proliferation of HepG.2.2.15 cells; and (B) knockdown of DDB1 in the normal liver cell line L02 resulted in reduced cell proliferation.

The HBV genome encodes four major viral RNA species: the more than full-length pregenomic RNA (pgRNA), which serves as the template for reverse transcription and encodes core protein and polymerase, as well as three subgenomic mRNAs necessary for the translation of the envelope proteins, and the mRNA for the $\mathrm{X}$ protein [27]. siRNAs specific for each of these transcripts have been used to suppress HBV expression [5-10]. HBx is associated with DDB1 [14,15], therefore, in this study, we focused on the concerted effect of HBx-siRNA and DDB1-siRNAs. However, knockdown of DDB1 specifically compromises the replication of HBV [19], therefore, knockdown of DDB1 may also enhance the antiviral activity of siRNAs targeting to other HBV transcripts.

\section{Acknowledgements}

We thank You-Lan Shan and Ya Luo for technical assistance. We are grateful to Dr. Wenjie Yang for reading this manuscript and providing guidance. This work was supported by Medical Science Foundation Project of Health Bureau of Chongqing Province (06-2-083) and NSFC (3070078 and 30671869).

\section{References}

[1] Kao JH, Chen DS. Global control of hepatitis B virus infection. Lancer Infect Dis 2002;2:395-403.

[2] Hanazaki K. Antiviral therapy for chronic hepatitis B: a review. Curr Drug Targets Inflamm Allergy 2004;3:63-70.

[3] Hannon GJ. RNA interference. Nature 2002;418:244-51.

[4] Fire A, Xu S, Montgomery MK, Kostas SA, Driver SE, Mello CC. Potent and specific genetic interference by double-stranded RNA in Caenorhabditis elegans. Nature 1998;391:806-11.

[5] Shlomai A, Shaul Y. Inhibition of hepatitis B virus expression and replication by RNA interference. Hepatology 2003;37:764-70.

[6] McCaffrey AP, Nakai H, Pandey K, Huang Z, Salazar FH, Xu H, et al. Inhibition of hepatitis B virus in mice by RNA interference. Nat Biotechnol 2003;21:639-44.

[7] Wu KL, Zhang X, Zhang J, Yang Y, Mu YX, Liu M, et al. Inhibition of Hepatitis B virus gene expression by single and dual small interfering RNA treatment. Virus Res 2005;112:100-7.

[8] Wu HL, Huang LR, Huang CC, Lai HL, Liu CJ, Huang YT, et al. RNA interference-mediated control of hepatitis $\mathrm{B}$ virus and emergence of resistant mutant. Gastroenterology 2005;128:708-16.

[9] Xuan B, Qian Z, Hong J, Huang W. EsiRNAs inhibit Hepatitis B virus replication in mice model more efficiently than synthesized siRNAs. Virus Res 2006;118:150-5.

[10] Morrissey DV, Lockridge JA, Shaw L, Blanchard K, Jensen K, Breen W, et al. Potent and persistent in vivo anti-HBV activity of chemically modified siRNAs. Nat Biotechnol 2005;23:1002-7.

[11] Tang H, Banks KE, Anderson AL, McLachlan A. Hepatitis B virus transcription and replication. Drug News Perspect 2001;14:325-34.

[12] Keeney S, Chang GJ, Linn S. Characterization of a human DNA damage binding protein implicated in xeroderma pigmentosum E. J Biol Chem 1993;268:21293-300.

[13] Keeney S, Eker AP, Brody T, Vermeulen W, Bootsma D, Hoeijmakers JH, et al. Correction of the DNA repair defect in xeroderma pigmentosum group $\mathrm{E}$ by injection of a DNA damage-binding protein. Proc Natl Acad Sci USA 1994;91:4053-6.

[14] Lee TH, Elledge SJ, Butel JS. Hepatitis B virus X protein interacts with a probable cellular DNA repair protein. J Virol 1995;69:1107-14.

[15] Bergametti F, Sitterlin D, Transy C. Turnover of hepatitis B virus X protein is regulated by damaged DNA-binding complex. J Virol 2002; 76:6495-501.

[16] Colgrove R, Simon G, Ganem D. Transcriptional activation of homologous and heterologous genes by the hepatitis $\mathrm{B}$ virus $\mathrm{X}$ gene product in cells permissive for viral replication. J Virol 1989;63:4019-26.

[17] Melegari M, Scaglioni PP, Wands JR. Cloning and characterization of a novel hepatitis $\mathrm{B}$ virus $\mathrm{X}$ binding protein that inhibits viral replication. J Virol 1998;72:1737-43.

[18] Klein NP, Bouchard MJ, Wang LH, Kobarg C, Schneider RJ. Src kinases involved in hepatitis B virus replication. EMBO J 1999;18:5019-27.

[19] Leupin O, Bontron S, Strubin M. Hepatitis B virus X protein stimulates viral genome replication via a DDB1-dependent pathway distinct from that leading to cell death. J Virol 2005;79:4238-45.

[20] Hu J, McCall CM, Ohta T, Xiong Y. Targeted ubiquitination of CDT1 by the DDB1-CUL4A-ROC1 ligase in response to DNA damage. Nat Cell Biol 2004;6:1003-9.

[21] Sells MA, Chen ML, Acs G. Production of hepatitis B virus particles in Hep G2 cells transfected with cloned hepatitis B virus DNA. Proc Natl Acad Sci USA 1987;84:1005-9.

[22] Wieland SF, Spangenberg HC, Thimme R, Purcell RH, Chisari FV. Expansion and contraction of the hepatitis B virus transcriptional template in infected chimpanzees. Proc Natl Acad Sci USA 2004;101:2129-34. 
[23] Chu G, Chang E. Xeroderma pigmentosum group E cells lack a nuclear factor that binds to damaged DNA. Science 1998;242:564-7.

[24] Wertz IE, O'Rourke KM, Zhang Z, Dornan D, Arnott D, Deshaies RJ, et al. Human de-etiolated-1 regulates c-Jun by assembling a CUL4A ubiquitin ligase. Science 2004;303:1371-4.

[25] Bontron S, Lin-Marq N, Strubin M. Hepatitis B virus X protein associated with UV-DDB1 induces cell death in the nucleus and is functionally antagonized by UV-DDB2. J Biol Chem 2002;277: 38847-54.

[26] Cang Y, Zhang J, Nicholas SA, Kim AL, Zhou P, Goff SP. DDB1 is essential for genomic stability in developing epidermis. Proc Natl Acad Sci 2007;104:2733-7.

[27] Seeger C, Mason WS. Hepatitis B virus biology. Microbiol Mol Biol Rev 2000;64:51-68. 